\title{
Special Edition on HFT2008 International Symposium
}

Established in 1961, the International Symposium series on Human Factors in Telecommunication (HFT) is organized once every two years to provide a forum for human factors experts, researchers and telecommunication design practitioners to discuss and share their experiences in the research and the application of human factors principles in telecommunication. Its interest includes the evaluation, design and application of human factors techniques and approaches for all aspects of the telecommunication and information and communication technology (ICT) industries on its policies, products, services, standards, and content development. The 21st Symposium on Human Factors in Telecommunication (HFT 2008) was hosted by the Interface Design Department at the Faculty of Creative Multimedia, Multimedia University, Malaysia, and this was the first time the HFT series of symposia was held in Asia.

HFT2008 was endorsed by the European Telecommunications Standards Institute (ETSI) Human Factors Group and it took place from the 17th until 20th of March, 2008 at the Renaissance Kuala Lumpur Hotel in Malaysia. Bridging industry and academia for over forty-six years, the HFT Symposium series continue to be guided by an international Permanent Steering Committee comprising of experts in the field of telecommunication, ICT and human factors.

This year's HFT2008 addressed the theme User Experience of ICTs, and in addition to paper presentations there were also workshops on guidelines for the generic user interface elements for $3 G$ mobile devices, services and applications, and personalization and user profile management. There were also poster presentations throughout the symposium. To reflect on the multidisciplinary nature of the symposium, the topics of interests were categorized as follows:

- Accessibility and Design for All

- Human Factors Methods and Techniques

- Mobile Communication

- Personalization and Profiling

- Networking and ICT Applications

- Standards and Frameworks

- User Interface Design

- Voice User Interface

The five papers that have been included in this special edition of International Journal of Interactive Mobile Technologies (iJIM) have been selected for three reasons. Firstly, these papers are of a high quality and reflect the scientific standards that one would expect at an international symposium. Furthermore, some of these papers were shortlisted for the John Karlin Best Paper Award at HFT2008. The authors were also given the opportunity to incorporate any comments raised at the symposium and any updates in their field of research and development which they feel would enhance the quality of their papers. Secondly, these papers reflect the current trend, diversity and interests in the field. The five papers reflect contributions from fourteen authors representing 
seven different countries. Finally, it is recognized that the journal, iJIM, is growing to be one of the leading journals in interactive mobile technologies, and it is an honor to be provided the opportunity to publish here. To this end, these papers have been selected due to their pertinence to issues prevalent in the fundamentals, applications, services and experiences in the field of interactive mobile technologies.

The paper by Bartolomeo, Petersen and Pluke highlights a growing interest in the telecommunication industry and service providers towards user personalization and profile management. This is especially so for Next Generation Networks and the potential benefits it offers to future mobile users and the multitude of user experiences that transacts between such networks. The paper also draws attention to the variety of research and development work being conducted at ETSI towards the standardization of system architecture and objects for profiles.

Mannonen looks at various cultural aspects of user interfaces, and its effect towards communication and collaborative tools and devices. Bringing together previous research work from 2004-2006, the paper looks at distributed and mobile knowledge workers for studying the role of information and communication technology towards them. A user interface culture analysis is suggested for promoting a better understanding of the users' preferences and approach towards using and applying communication technology.

Useful insights for the legibility of characters on a liquid crystal display for the aged, especially towards short messaging services (SMS), is presented in a paper by Hasegawa, Miyao, Matsunuma, Fujikake and Omori. A study was conducted making reference to the ISO 9241-3 on recommendations for viewing height and distances. A recommendation is provided for applying universal design considerations for the design of mobile content, devices and applications so as not to leave out the elderly users.

Wong, Khong and Thwaites describes the application of a user interface design process model on a mobile community project for the hearing impaired. Taking an iterative design process model as a generic benchmark, the paper establishes successive layers of user-centered techniques to the model. The authors leverage on existing usability and universal design methods for approaching designing a mobile community project for the hearing impaired society.

The paper by Nyberg and Chincholle presents how MSN Messenger is still a complement (or not) to mobile users. Instant messenger applications and its supporting services are a fast growing trend in the mobile service industry. The paper stems from research work carried out by Ericsson Research in 2007 followed by a more recent work nearly a year later in April 2008, thus updating the contents of the original paper submitted to HFT2008. The work relates to Swedish mobile MSN users and move towards comparing its significance with SMS. 
We wish to express our appreciation to our Platinum Sponsor, Telenor, the European Telecommunication Standards Institute (ETSI), and all of HFT2008's strategic partners for their support. Our gratitude also goes to the keynote speakers, Kristin Braa (Vice President of Internationalization, Telenor), Takahiro lijima (Team Leader of Human Interaction Design, Director and Manager, Panasonic Design Company) and Jim Nieters (Director of User Experience, Yahoo! Inc.) for their invaluable contributions, generosity and participation. Our sincere thanks also go to the Dean of the Faculty of Creative Multimedia at Multimedia University, Associate Professor Harold Thwaites, the Local Organizing Members, June, Anthony, Asyraf, Kimberly and Dr. Quek, and to all the student volunteers who assisted. Last but not least, we want to thank the symposium's international Permanent Steering Committee, especially Bruno, Martin and Ed, who were with us during the event, and to everyone else who helped make HFT2008 a memorable event in Asia.

\section{Chee Weng Khong and Chui Yin Wong}

Interface Design Department, Faculty of Creative Multimedia, Multimedia University,

Cyberjaya, Malaysia.

http://www.hft2008.org 